\title{
TEXTURE ANALYSIS FOR CLASSIFICATION OF RISAT-II IMAGES
}

\author{
D. Chakraborty ${ }^{\mathrm{a}^{*}}, \mathrm{~S}$ Thakur ${ }^{\mathrm{b}}$, A Jeyaram $^{\mathrm{a}}, \mathrm{YVN}_{\mathrm{K}}$ Krishna Murthy $^{\mathrm{c}}, \mathrm{VK}$ Dadhwal $^{\mathrm{c}}$ \\ ${ }^{a}$ Regional Remote Sensing Centre - East, NRSC, ISRO, Kolkata, India (deba.isro@gmail.com) \\ ${ }^{\mathrm{b}}$ Sikkim Manipal Institute of Technology, Sikkim, India \\ ${ }^{\mathrm{c}} \mathrm{NRSC}$, ISRO, Hyderabad, India
}

\section{Commission III, Working Group ICWG III/VII}

\author{
KEYWORDS: Image, Texture, Method, Interpretation, Analysis, Development and Classification
}

\begin{abstract}
RISAT-II or Radar Imaging satellite - II is a microwave-imaging satellite lunched by ISRO to take images of the earth during day and night as well as all weather condition. This satellite enhances the ISRO's capability for disaster management application together with forestry, agricultural, urban and oceanographic applications. The conventional pixel based classification technique cannot classify these type of images since it do not take into account the texture information of the image. This paper presents a method to classify the high-resolution RISAT-II microwave images based on texture analysis. It suppress the speckle noise from the microwave image before analysis the texture of the image since speckle is essentially a form of noise, which degrades the quality of an image; make interpretation (visual or digital) more difficult. A local adaptive median filter is developed that uses local statistics to detect the speckle noise of microwave image and to replace it with a local median value. Local Binary Pattern (LBP) operator is proposed to measure the texture around each pixel of the speckle suppressed microwave image. It considers a series of circles (2D) centered on the pixel with incremental radius values and the intersected pixels on the perimeter of the circles of radius $r$ (where $r=1,3$ and 5 ) are used for measuring the LBP of the center pixel. The significance of LBP is that it measure the texture around each pixel of the image and computationally simple. ISODATA method is used to cluster the transformed LBP image. The proposed method adequately classifies RISAT-II X band microwave images without human intervention.
\end{abstract}

\section{$1.0 \quad$ INTRODUCTION}

Optical remote sensing technology is used for mapping the earth surface. But it cannot map the earth surface in all weather conditions since optical bands cannot penetrate clouds, smog and haze. Microwave remote sensing technology is used as an alternative technology for mapping the earth surface features especially when optical data is not available (Oliver et al., 1998). But interpretation of microwave images is very difficult due to the presence of texture in these images. Classical methods namely K-Means (Hartigan et al. 1979), Fuzzy C Means (Bezdek et al. 1984) and methods of Minimum Distance (Richards, 1995) are not adapted because they fail to take into account the fact that a texture can be represented as a mix of smooth regions and very sharp transitions. The issue of texture based image classification is an old and difficult problem, which is still a field of a lot of research.

Relevant studies on Texture Classification: GLCM (Gray Level Co-occurrence matrix) algorithm (Tsai et al. 2006; Clausi et al. 2004; Tsai et al. 2005; Haralick et al. 1973) quantifies the texture by measuring the spatial frequency of co-occurrence of pixel gray levels in a user defined moving kernel (window). Since the size of a GLCM matrix depends on the data range of pixel gray values, images of large numbers of data bits may result in large matrix sizes during GLCM operation and require a large amount of memory and CPU cycles to handle the computation. The reductions of GLCM matrix sizes by rescaling the image gray levels to a lower data bit number reduce the classification accuracy. Another important factor that may cause substantial impact to GLCM processing is the kernel size. Moreover, the method is not able to properly identify the boundary region between the textures.

Markov random field (MRF) model (Szira yi et al. 2000; Clausi et al. 2004; Chellappa et al. 1985; Solberg et al. 1996; Chen et al. 1998) assumes that the intensity at each pixel in the image depends on the intensities of the neighboring pixels. The method segment the image based on the local calculations of probability and potential functions. The draw back of the method is that, it cannot properly handle the data from classes not present in the training set, erodes the quality of the segmentation in the boundary region and needs huge amount of computing power.

Some more techniques like the gamma, Weibull, and $\mathrm{K}$ distributions have been used to classify microwave images (Bernad et al. 2009; Dong et al. 2001; Tison et al. 2004; Petrou et al. 2002). These mathematical models have done well in characterizing low-resolution microwave images. But most of them failed to classify high-resolution microwave images due to presence of complex structure of textures in such type of images. RISAT II is capable of high-resolution microwave imaging of $1 \mathrm{~m}, 3 \mathrm{~m}, 1.8 \mathrm{~m}$ and $8 \mathrm{~m}$. Therefore classification of this type of high-resolution microwave images without much human intervention is required to be developed. 


\section{Local Binary Pattern (LBP)}

In last two decades Local Binary Pattern has been used for texture analysis of images including remote sensing images [Ojala et al. 1996; Ojala et al. 2002; Lucieer et al. 2005]. The central concept of LBP is that it measures the gray-scale invariant texture of the image. It is derived from the analysis of a $3 \times 3$ local neighborhood over a central pixel. The LBP is based on a binary code describing the local texture pattern. This code is built by thresholding a local neighborhood by the grey value of its center. The eight neighbors are labeled using a binary code $\{0,1\}$ obtained by comparing their values to the central pixel value. If the tested grey value is below the grey value of the central pixel, then it is labeled 0 , otherwise it is assigned the value 1 . The obtained value is then multiplied by the weights given to the corresponding pixels. The weight is given by the value $2^{i+1}$. Summing the obtained values gives the measure of the LBP. The microwave images contain texture and non-texture region. Advantages of using LBP on microwave images are that (i) it can be used as a tool to measure the spatial pattern around each pixel of the image and (ii) it does not require any prior information about the pixel intensity, (iii) LBP technique theoretically and computationally is simple. This work attempts to describe the textures of microwave images using LBP.

\section{$1.2 \quad$ Objective}

The aim of this study is to develop a method to classify high resolution microwave images. To achieve this aim (i) local adaptive median filter is developed to replace the speckle noise of microwave images with a local median value, (ii) LBP is used as a tool to measure the texture around each pixel of the speckle suppressed image and (iii) ISODATA clustering technique is used to cluster the transformed LBP image.

\subsection{Outline of the Proposed Work}

A median filter is developed to suppress the speckle noise from microwave image. A new method is proposed to compute the LBP of pixels in the image. This LBP analysis is used to measure the texture around each pixel of the noise suppressed image. To show the robustness of the proposed LBP analysis the ISODATA is applied separately on the transformed images obtained from Lucieer et al's LBP analysis as well as images obtained from the proposed LBP analysis and compared the results. From the results, it is found that the "Lucieer et al's LBP analysis and ISODATA" under segment the images where as "proposed LBP analysis and ISODATA" segment classes distinctly. The proposed method is proved to be effective to classify RISAT-II microwave images.

The paper is organized as follows. Section 2.0 discusses about methodology and consists of three sub-sections. Sub-section 2.1 discusses the methods used for speckle suppression. Subsection 2.2 discusses the methods used for Image Transformation. Sub-section 2.3 discusses about the clustering of transformed image. Comparison of the result of "Lucieer et al's LBP analysis and ISODATA" and "Proposed LBP analysis and ISODATA" is discussed in Section 2.4. The final conclusions are drawn in Section 3.0.

\subsection{METHODS}

Methodology adapted to classify the microwave images has three main steps: (i) suppression of speckle noise, (ii) transformation and (iii) clustering. In the first step speckle is identified from microwave image by using local statistics and replaced it with a local median value. In the second step each pixel of the image is transformed into degree of texture based on the spatial pattern of the neighborhood. In the third step, ISODATA clustering technique is used to cluster the transformed image.

\subsection{Speckle Suppression}

A local adaptive median filter is developed to suppress the speckle noise from microwave image. It is two step procedures: (i) in the first step speckle is identified from microwave image and (ii) identified speckle is suppressed in the second step. The central pixel value that is not falling in between the range of lower bound (LB) and upper bound (UB) value is considered as a speckle. LB and UB are represented here as $(\mu-\sigma)$ and $(\mu+$ $\sigma)$ respectively, where $\mu$ and $\sigma$ stand for mean and standard deviation of the pixel values falling in the local window respectively. The pixel value of the central pixel position of the local window that is not falling in between LB and UB replaced by the median (M) of the pixel values of local window.

\section{$2.2 \quad$ Transformation}

Transformation is carried out on the speckle-suppressed image. The LBP is used as a tool to transform the image. Intersected pixels by the perimeter of the circles are used for computing the LBP. The proposed method uses a series of circles (2D) centered on the pixel with incremental radius values. Measures rotation invariant texture for each circularly symmetric neighborhood. Finally all measure together becomes LBP value of the center pixel of the kernel. The significance of uses of a series of circles is that (i) the circular neighborhoods enable a definition of a rotation invariant texture and (ii) multiple circles facilitate to consider more number of pixels than a circle to compute the degree of texture of the neighbor of the center pixel of the kernel. Detail procedure to compute the degree of texture of the neighborhood of the pixel is given bellow:

Initially for each pixel a series of three values of radius $r$ (where $\mathrm{r}=1,3$ and 5) are considered as shown in figure 1 for measuring the degree of texture around the pixel.

The grey values $\left\{\mathrm{f}_{0}, \mathrm{f}_{1}, \ldots \ldots \ldots \ldots, \mathrm{f}_{\mathrm{P} 1-1}\right\},\left\{\mathrm{g}_{0}, \mathrm{~g}_{1}, \ldots \ldots \ldots \ldots\right.$, $\left.\mathrm{g}_{\mathrm{P} 2-1}\right\}$ and $\left\{\mathrm{h}_{0}, \mathrm{~h}_{1}, \ldots \ldots \ldots \ldots, \mathrm{h}_{\mathrm{P} 5-1}\right\}$ of intersected pixels on the perimeter of the circle of radius $r=1, r=3$ and $r=5$ respectively are used for measuring the same. Here, $\mathrm{P}_{1}\left(\mathrm{P}_{1}>1\right)$, $\mathrm{P}_{2}\left(\mathrm{P}_{2}>1\right)$ and $\mathrm{P}_{3}\left(\mathrm{P}_{3}>1\right)$ are the total number of intersected pixels on the perimeter of the circle of radius $r=1, r=3$ and $r=5$ 
International Archives of the Photogrammetry, Remote Sensing and Spatial Information Sciences, Volume XXXIX-B3, 2012 XXII ISPRS Congress, 25 August - 01 September 2012, Melbourne, Australia

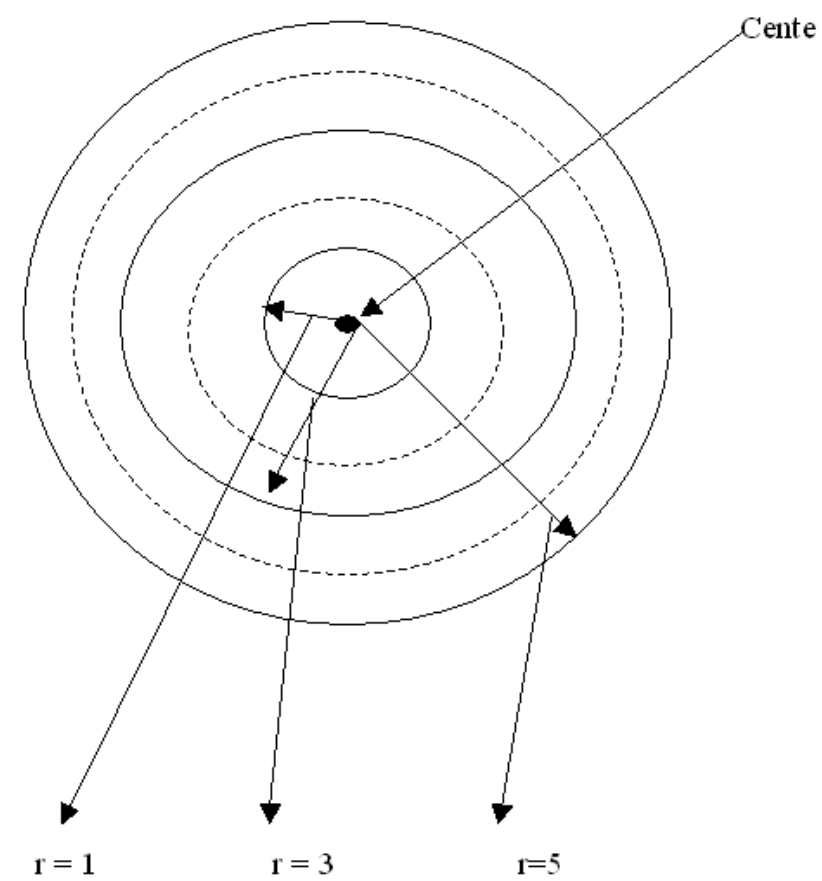

Figure 1: Circles of increasing $r=1,3$ and 5 radius for measuring degree of texture around each pixel of the image.

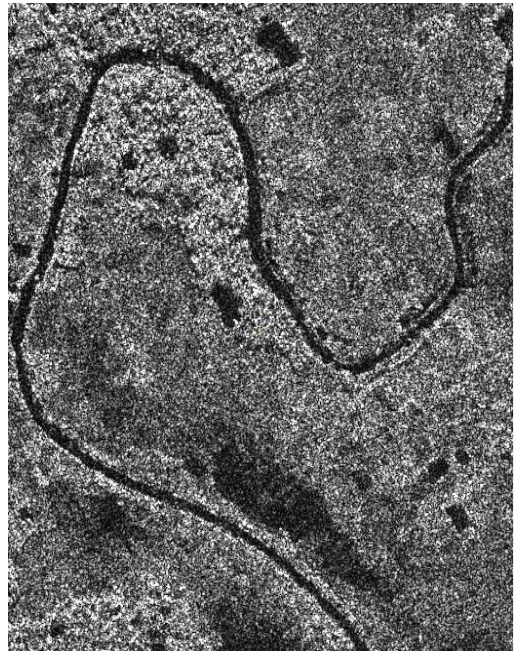

a

Water body
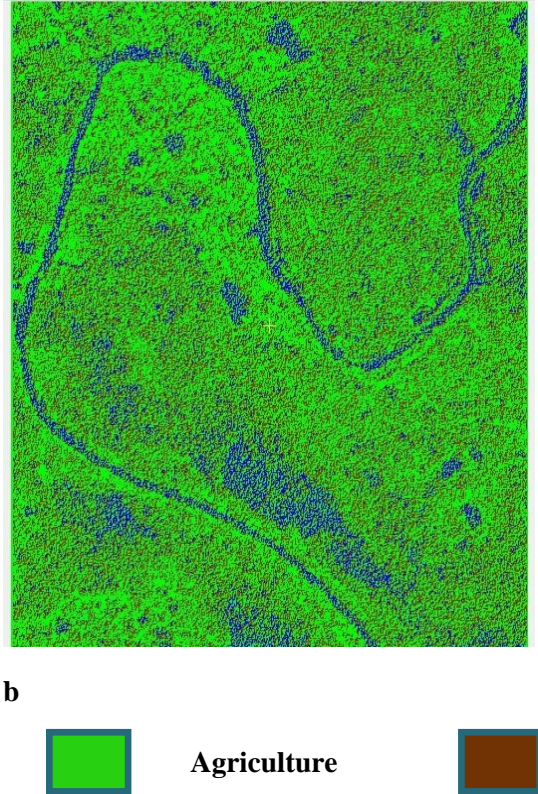

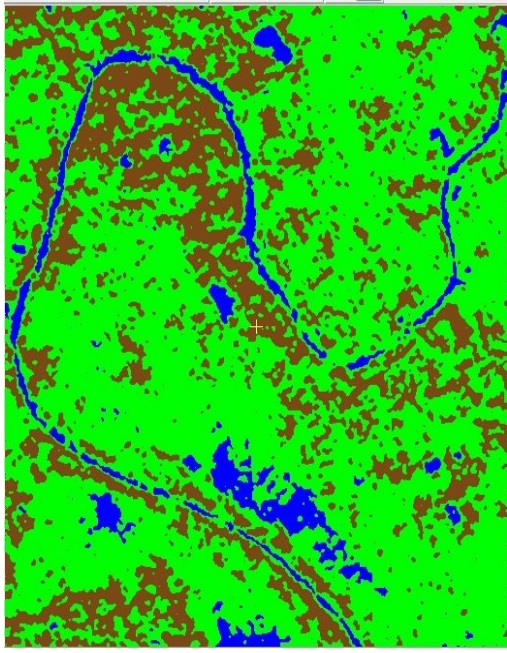

c

Built-up area

Figure 2a-c:

(a) RISAT-II image of agriculture, built-up area and water bodies,

(b) Output image obtained by applying "Lucieer et al's LBP analysis and ISODATA" on (a),

(c) Output image obtained by applying "proposed LBP and ISODATA" on (a). 
respectively. With the center pixel as the threshold, its neighbors (i.e. intersected pixels) are labeled as 1 (where digital number of the neighborhood is larger than that of the center) or 0 (where digital number of the neighborhood is smaller than that of the center). Consequently the number of 1 to 0 or 0 to 1 transition $\xi_{\mathrm{r}=1}, \xi_{\mathrm{r}=3}$ and $\xi_{\mathrm{r}=5}$ for the circle of radius $\mathrm{r}=1, \mathrm{r}=3$ and $\mathrm{r}=5$ are computed respectively as follows:

$$
\begin{aligned}
& \xi_{r=1}=\sum_{i=1}^{P_{1}}\left|s\left(f_{i}-f_{c}\right)-s\left(f_{i-1}-f_{c}\right)\right| \\
& \xi_{r=3}=\sum_{j=1}^{P_{3}}\left|s\left(g_{j}-g_{c}\right)-s\left(g_{j-1}-g_{c}\right)\right| \\
& \xi_{r=5}=\sum_{k=1}^{P_{5}}\left|s\left(h_{k}-g_{c}\right)-s\left(h_{k-1}-h_{c}\right)\right|
\end{aligned}
$$

Where,

$\mathrm{P}_{1}$ is the intersected pixels on the perimeter of the circle of radius $r=1$

$\mathrm{P}_{3}$ is the intersected pixels on the perimeter of the circle of radius $r=3$,

$\mathrm{P}_{5}$ is the intersected pixels on the perimeter of the circle of radius $\mathrm{r}=5$.

$f_{c}, g_{c}, h_{c}$ is the grey values of the center pixel $\left(p_{c}\right)$ and $f_{c}=g_{c}=h_{c}$ and

$\mathrm{S}(\mathrm{x})=\left\{\begin{array}{l}1, \mathrm{x} \geq 0 \\ 0, \mathrm{x}<0\end{array}\right.$

Finally the total transition $\xi_{\text {Tot }}$ is calculated as follows:

$$
\xi_{\text {Tot }}=\Sigma \xi_{\mathrm{r}}, \mathrm{r}=1,3,5
$$

Transition $\xi_{\text {Tot }}$ is considered as the LBP value of the center pixel. The above arrangement is moved over the whole image until all pixels considered. As a result the original image ' $\mathrm{I}$ ' is transformed into degree of texture on the basis of its neighbor. The transformed image is represented here as $\mathrm{I}^{\mathrm{LBP}}$. The presented method for selecting the texture feature value using 1 to 0 or 0 to 1 transitions retains the rotation invariance of the texture measurement system, since, the number of transitions do not change if the texture is rotated.

\subsection{Clustering}

The Interactive Self-Organizing Data Analysis Technique (ISODATA) method (Jain et al. 1999; Kohei et al. 2007) is used to cluster transformed image. It includes three key steps. First, assign some arbitrary clustering centers in the image. Next, classify each pixel to the nearest cluster. Last, calculate all the new cluster centers on the basis of every pixel in one cluster set. Step 2 and step 3 are iterative and they stop until the change between two iterations is fine or little. During each iteration the ISODATA clustering algorithm may have refinement by splitting or merging clusters. Clusters are merged if either the number of members (pixel) in a cluster is less than a certain threshold or if the centers of two clusters are closer than a certain threshold. Clusters are split into two different clusters if the cluster standard deviation exceeds a predefined value and the number of members (pixels) is twice the threshold for the minimum number of members. ISODATA clustering algorithm has many benefits such as less computing, fast computing speed and simplicity as well as un-supervising.

\section{$2.4 \quad$ Results and Discussion}

"Lucieer et al's LBP analysis and ISODATA" and "Proposed LBP analysis and ISODATA" have been applied on a $3 \mathrm{~m}$ spatial resolution RISAT-II X band microwave image (shown in Figure 2a ) of (i) vegetation, (ii) built-up area, and (iii) water bodies. Texture is visible in the images. The results of "Proposed LBP analysis and ISODATA" method is then compared with the results obtained from the analysis based on "Lucieer et al's LBP analysis and ISODATA" respectively.

The "Lucieer et al's LBP analysis and ISODATA" and "Proposed LBP analysis and ISODATA" methods are applied on a RISAT-II X-band microwave image are shown in Figure $2 b$ and $2 c$. In the output images i.e. in Figure $2 b$ and $2 c$ green, blue and brown colors represents agriculture, water bodies and built-up area respectively. From the results, it clearly appears that the "Lucieer et al's LBP analysis and ISODATA" method gives heterogeneous segments. While " Proposed LBP analysis and ISODATA" method gives more homogeneous segments with distinct classes than "Lucieer et al's LBP analysis and ISODATA" method.

Using the ground truth data overlaid separately on the resultant outputs obtained from "Lucieer et al's LBP analysis and ISODATA" and "Proposed LBP analysis and ISODATA" methods, the area statistics of the classification rates for each approach is shown in Table 1. The numerical results shows that the success rate for recognizing agriculture, built-up area and Water bodies are $(48.48,12.23,58.36)$ by "Lucieer et al's LBP analysis and ISODATA" whereas (82.55, 73.65 and 86.20 ) by the "Proposed LBP analysis and ISODATA" approach. 


\begin{tabular}{|c|c|c|c|c|}
\hline \multirow{2}{*}{ Classification Method } & \multirow{2}{*}{$\begin{array}{l}\% \text { Area occupied } \\
\text { by the class as } \\
\text { per classification } \\
\text { results }\end{array}$} & \multicolumn{3}{|c|}{ Identified ground truth features } \\
\hline & & $\begin{array}{c}\text { Agriculture } \\
(100 \%)\end{array}$ & $\begin{array}{c}\text { Built-up area } \\
(100 \%)\end{array}$ & $\begin{array}{l}\text { Water bodies } \\
(100 \%)\end{array}$ \\
\hline \multirow{3}{*}{$\begin{array}{l}\text { Lucieer et al's } \\
\text { LBP analysis and } \\
\text { ISODATA } \\
\text { (Figure } 2 b \text { ) }\end{array}$} & Agriculture & 48.48 & 78.26 & 41.64 \\
\hline & Built-up area & 36.83 & 12.23 & - \\
\hline & Water bodies & 14.69 & 9.51 & 58.36 \\
\hline \multirow{3}{*}{$\begin{array}{l}\text { Proposed LBP } \\
\text { analysis and } \\
\text { ISODATA } \\
\text { (Figure 2c) }\end{array}$} & Agriculture & 82.55 & 19.16 & 13.8 \\
\hline & Built-up area & 13.66 & 73.65 & - \\
\hline & Water bodies & 3.79 & 7.19 & 86.20 \\
\hline
\end{tabular}

Table 1: The comparative success rate for classifying the features obtained by applying "Lucieer et al's LBP analysis and ISODATA" and "Proposed LBP analysis and ISODATA" separately on RISAT-II X-Band image. The column of the Table represents the percentage (\%) of area occupied by the features according to the classification results when there is a unique feature as per the ground truth.

The experimented results with the input image (Figure 2a) shows that the "Lucieer et al's LBP analysis and ISODATA" technique under segment (i) agriculture area mixed with builtup area, (ii) water bodies mixed with the agriculture shown in Figure $2 \mathrm{~b}$. This discrepancy decreases the success rate of recognizing agriculture, built-up area and water bodies as shown in Table 1. The "Proposed LBP analysis and ISODATA" mostly overcome these discrepancies. It is found that the superposition of agriculture, water bodies, and built-up area becomes less as shown in Figure 2c. Moreover the decreased discrepancies increase the success rate in recognizing agriculture, water bodies and built-up area (shown in Table 1).

2.4.1 Comparison between Lucieer et al's [2005] classification technique and proposed technique

- Lucieer et al's employ a circle of fixed radius 'c' from the center pixel position of the kernel and intersected pixels on the perimeter of that circle are only considered for measuring the texture around that pixel. As a result most of the pixels of the kernel are not used for measuring the texture. The proposed method uses a series of circles (2D) centered on the pixel with incremental radius values. The intersected pixels on the perimeter of the circles of radius $r$ (where $r=1,3$ and 5) are considered for measuring the texture around that pixel. As a result it uses more number of pixels of the kernel for measuring the texture around each pixel of the image.

- Lucieer et al's used supervised (fuzzy c-means) classification technique where as the proposed technique an un-supervised (ISODATA) classification technique.
- The experimental results with the input image (Figure 2a) shows that the use of "Lucieer et al's" technique can superpose regions namely built-up area and agriculture as shown in Figure 2b. The proposed technique mostly overcomes these discrepancies as shown in Figure 2c.

\subsection{CONCLUSIONS}

In this paper LAM (Local Adaptive Median) filter is developed to suppress the speckle noise from RISAT-II image. LBP is used as a tool to compute the degree of texture around each pixel in the microwave image. This computed texture measure around each pixel in the image is used farther to classify the microwave image. From the results of the experiments it is found that the proposed method adequately clusters complex images containing texture region as well as non-texture region. Moreover it can be considered as an intuitively appealing, unsupervised (no need for a predefinition of the number of clusters) and fast clustering algorithm. As a result the method is potentially useful to classify RISAT-II microwave images efficiently and accurately.

\subsection{REFERENCES}

Bernad, P., Denise, G., and Réfrégier, R., 2009. Hierarchical feature-based classification approach for fast and userinteractive SAR image interpretation, IEEE Geosci. Remote Sensing Letter. 6(1), pp. 117-121.

Bezdek J C., Ehrlich R and Full W., 1984. FCM: the Fuzzy cMeans clustering algorithm, Computers and Geosciences, 10, pp. 191-203. 
Clausi, D A. and Yue, B., 2004. Texture Segmentation Comparison Using Grey Level Co-occurrence Probabilities and Markov Random Fields, Proceedings of the 17th International Conference on Pattern Recognition (ICPR'04); Available at: http://ieeexplore.ieee.org/stamp/.

Chellappa, R. and Chatterjee, S., 1985. Classification of Textures Using Gaussian Markov Random Fields, IEEE Transactions on Acoustics, Speech, and Signal Processing, 33, pp. 959-963.

Chen, C H., Pau, L F. and Wang, P S P., 1998. The Handbook of Pattern Recognition and Computer Vision (2nd Edition), by World Scientific Publishing Co., pp. 207-248.

Dong, Y., Milne, A. K., and Forster, B. C., 2001. Segmentation and classification of vegetated areas using polarimetric SAR image data, IEEE Trans Geosci. Remote Sens., 39 (2), pp. $321-$ 329.

Hartigan, J.. A., and Wong, M. A., 1979. A K-means clustering algorithm; Appl. Stat.. 28(1), pp. 100-108.

Haralick, R..M., Shanmugan, K., and Dinstein, I.,1973. Textural features for image classification, IEEE Transactions on systems, Man, and cybernetics, 3 (6), pp. 610-621.

Jain, A. K., Murty, M. N., Flynn, P. J., 1999. Data clustering: a review, ACM Computing Surveys, 31(3), pp. 264-323.

Kohei, A., and XianQiang, Bu., 2007. ISODATA clustering with parameter (threshold for merge and split) estimation based on GA: Genetic Algorithm, Report Facalty Science Engineering, Saga University, 36 (1), pp. 17-23 ( available at http://dlwww.dl.saga-

u.ac.jp/contents/mgzn/ZR00005459/ZR00005459.pdf as on January 17, 2011).

Lucieer, A., Stein A., and Fisher, P., 2005. 'Texture-based segmentation of high-resolution remotely sensed imagery for identification of fuzzy objects', International Journal of Remote Sensing, 26(14), pp. 2917-2936

Oliver, C. J., and Quegan, S., 1998. Understanding Synthetic Aperture Radar Images, New York: Artech House.

Ojala, T.P.M., Harwood, D., 1996. A comparative study of texture measures with classification based on featured distribution. Pattern Recognition, 29(1), pp. 51-59.

Ojala, T., Pietikäinen, M., and Mäenpää, T., 2002. Multiresolution gray-scale and rotation invariant texture classification with local binary patterns. IEEE Transactions on Pattern Analysis and Machine Intelligence, 24, pp. 971-987.

Petrou, M., Giorgini, F., and Smits, P., 2002. Modelling the histograms of various classes in SAR images, Pattern Recognit. Lett., 23(9), pp. 1103-1107.

Richards, J. A., 1995. Remote Sensing Digital Image Analysis:An Introduction, Springer-Verlag, pp. 265-290

Szira yi, T., Zerubia, J., Czu' I, L., Geldreich, D. and Kato, Z., 2000. Image Segmentation Using Markov Random Field Model in Fully Parallel Cellular Network Architectures, Real-Time Imaging, 6, pp. 195-211.
Solberg, A. H. S. S., and Jain, A. K., 1996. A Markov Random Field Model for Classification of Multisource Satellite Imagery, IEEE Transactions On Geoscience And Remote Sensing, 34(1).

Tsai, F. and Chou M J., 2006. Texture Augmented Analysis Of High Resolution Satellite Imagery In Detecting Invasive Plant Species, Journal of the Chinese Institute of Engineers, 29(4), pp. 581-592.

Tsai, F., Chou, M. J. and Wang, H. H., 2005. Texture Analysis of High Resolution Satellite Imagery for Mapping Invasive Plants, IEEE International Geoscience and Remote Sensing Symposium Proceedings, 4, pp. 3024- 3027.

Tison, C., Nicolas, J. M., Tupin, F., and Maître, H., 2004. A new statistical model for Markovian classification of urban areas in high-resolution SAR images, IEEE Trans. Geosci. Remote Sens., 42(10), pp. 2046-2057. 